\title{
Apoptosis in the skeletal muscle of patients with heart failure: investigation of clinical and biochemical changes
}

G Vescovo, M Volterrani, R Zennaro, M Sandri, C Ceconi, R Lorusso, R Ferrari, G B Ambrosio, L Dalla Libera

\begin{abstract}
Objective-To investigate the contribution of apoptosis in the development of the skeletal myopathy in chronic heart failure.

Design-The electrophoretic pattern of myosin heavy chains (MHC), fibre cross sectional area, number of in situ nick end labelling (TUNEL) positive apoptotic myocyte nuclei, and the tissue levels of caspase-3, Bcl-2, and ubiquitin were determined in biopsies taken from the vastus lateralis muscle. The study involved nine patients with severe chronic heart failure caused by ischaemic heart disease and hibernating myocardium and five controls.

Results-In chronic heart failure patients the vastus lateralis showed a significant increase of $\mathrm{MHC}_{2 \mathrm{a}}$ and $\mathrm{MHC}_{2 \mathrm{~b}}$ and a greater degree of fibre atrophy, as demonstrated by the decreased cross sectional area. There was also an increased number of TUNEL positive apoptotic myocyte nuclei. Tissue concentrations of $\mathrm{Bcl}-2$ were decreased, while those of caspase- 3 and ubiquitin were increased. Peak oxygen consumption $\left(\mathrm{VO}_{2}\right)$ was negatively correlated with the number of TUNEL positive nuclei and the fibre cross sectional area. There was a correlation between the number of apoptotic nuclei and the fibre cross sectional area, but no correlation between myosin heavy chains and number of apoptotic nuclei.

Conclusions-Myocyte apoptosis occurs in the skeletal muscle of patients with chronic heart failure, and its magnitude is associated with the severity of exercise capacity limitation and the degree of muscle atrophy. Muscle atrophy contributes to the limitation of exercise capacity, together with the increased synthesis of fast, more fatiguable myosin heavy chains.

(Heart 2000;84:431-437)
\end{abstract}

Keywords: apoptosis; chronic heart failure; exercise capacity; myosin heavy chains

Chronic heart failure is a clinical syndrome characterised by decreased exercise capacity caused by the early occurrence of fatigue and dyspnoea. ${ }^{1-8}$ There are several theories about the origin of these symptoms, although the contribution of intrinsic skeletal muscle abnormalities, with shift from the "slow" fatigue resistant type I to the more fatiguable "fast" type II fibres, is widely recognised..$^{9-12}$ However, the pathophysiology of this myopathy is still poorly understood. Triggers for changes in fibre type and their relation to muscle atrophy are obscure, and whether atrophy by itself contributes to the decreased exercise capacity is still debated. ${ }^{13-16}$ We have recently shown in man that the myosin heavy chain (MHC) composition of the leg skeletal muscle contributes to the limitation of exercise capacity, as peak oxygen consumption $\left(\mathrm{VO}_{2}\right)$ correlates positively with the expression of the slow fatigue resistant $\mathrm{MHC}_{1}$, and the improvements in $\Delta \mathrm{VO}_{2}$ after six months of pharmacological treatment correlate with the magnitude of changes in $\mathrm{MHC}_{1} \cdot{ }^{13} \mathrm{In}$ an experimental model of chronic heart failure we have recently shown that changes in myosin heavy chain composition are not secondary to muscle atrophy. Atrophy correlates with the magnitude of skeletal muscle apoptosis, which is likely to be triggered by circulating concentrations of tumour necrosis factor $\alpha(\mathrm{TNF} \alpha) .{ }^{14}$

Our aim in this study was to investigate the role of apoptosis in producing muscle atrophy and changes in fibre type in severe chronic heart failure in the human. This was done by looking at the relation between exercise capacity, changes in myosin heavy chains, degree of fibre atrophy, and myocyte and interstitial apoptosis.

\section{Methods}

The study was approved by the ethics committee of the University of Brescia, and written informed consent was obtained.

PATIENTS

We studied nine male patients with severe chronic heart failure who underwent coronary revascularisation. Their details are given in table 1. They were all diagnosed as having ischaemic heart disease and hibernating myocardium by coronary angiography, dobutamine echocardiography, exercise thallium ${ }^{201}$ scan, and deoxyglucose positron emission tomography. All underwent maximum symptom limited cardiopulmonary exercise testing with a modified Naughton protocol. A Schiller Cardiovit CS100 (Baar, Switzerland) with 1308 capnograph was used. Oxygen consumption at maximum exercise was expressed as peak oxygen consumption (peak $\mathrm{VO}_{2}$ ) defined as the mean oxygen consumption of the last 30 seconds of an incremental exercise test. In all the patients the ejection fraction and the left ventricular end systolic and end diastolic 
Table 1 Patient characteristics

\begin{tabular}{|c|c|c|c|c|c|c|c|c|c|c|c|c|c|c|c|c|}
\hline & Sex & $\begin{array}{l}\text { Age } \\
\text { (years) }\end{array}$ & Diagnosis & $\begin{array}{l}\text { MI } \\
\text { (years) }\end{array}$ & $\begin{array}{l}\text { CHF } \\
\text { (months) }\end{array}$ & $\begin{array}{l}\text { Diur } \\
\text { score }\end{array}$ & NYHA & $E F$ & $L V E D V$ & LVESV & $\mathrm{VO}_{2}$ & $M H C_{1}$ & $\begin{array}{l}\text { Interst } \\
\text { apopt }\end{array}$ & $\begin{array}{l}\text { Myo } \\
\text { apopt }\end{array}$ & Fatig & $\begin{array}{l}\text { Fibre } \\
C S A\end{array}$ \\
\hline 1 & $M$ & 45 & CAD & Y 5 & 3 & 3 & 3 & 24 & 190 & 144 & 15.6 & 62 & 15 & 0 & 81 & 5168 \\
\hline 2 & $M$ & 44 & CAD & Y 2 & 2 & 4 & 4 & 36 & 117 & 75 & 11.1 & 55 & 111 & 49 & 59 & 2094 \\
\hline 3 & M & 66 & CAD & Y 11 & 1 & 4 & 3 & 26 & 227 & 168 & 13.9 & 49 & 27 & 5 & 66 & 3743 \\
\hline 4 & $M$ & 72 & CAD & Y 10 & 1 & 2 & $2 b$ & 15 & 150 & 128 & 12.3 & 40 & 8 & 4 & 71 & 3368 \\
\hline 5 & M & 56 & CAD & Y 3 & 3 & 3 & 4 & 30 & 140 & 100 & 14.6 & 78 & 3 & 0 & 73 & 5196 \\
\hline 6 & $M$ & 58 & CAD & No & 2 & 5 & $2 b$ & 16 & 300 & 251 & 11.2 & 61 & 39 & 44 & 68 & 3944 \\
\hline 7 & $M$ & 41 & CAD & No & 9 & 1 & $2 b$ & 25 & 171 & 132 & 12.1 & 36 & 36 & 20 & 79 & 4182 \\
\hline 8 & M & 59 & CAD & Y 13 & 3 & 1 & 3 & 31 & 196 & 175 & 14.4 & 77 & 14 & 10 & 79 & 3250 \\
\hline 9 & M & 73 & CAD & No & 3 & 1 & $2 b$ & 14 & 228 & 196 & 11.1 & 68 & 50 & 37 & 56 & 2787 \\
\hline
\end{tabular}

$\mathrm{CAD}$, coronary artery disease; $\mathrm{CHF}$, duration of chronic heart failure (months); Diur score, diuretic score: 1, thiazide only; 2, 20-40 mg frusemide (furosemide); 3, $40-80 \mathrm{mg}$ frusemide; $4,80-120 \mathrm{mg}$ frusemide; $5, \geqslant 120 \mathrm{mg}$ frusemide; EF, ejection fraction (\%); Fatig, fatiguability as \% of initial maximum strength after 5 min exercise; Fibre CSA, myofibre cross sectional area $\left(\mu \mathrm{m}^{2}\right)$; Interst apopt, number of TUNEL positive interstitial nuclei/mm ${ }^{3}$; LVEDV, left ventricular end diastolic volume (ml); LVESV, left ventricular end systolic volume (ml); HHC $_{1}$, per cent distribution of myosin heavy chain 1 (\%); MI, myocardial infarction; Myo apopt, number of TUNEL positive myocyte nuclei $/ \mathrm{mm}^{3} ; \mathrm{Vo}_{2}$, peak oxygen consumption $(\mathrm{ml} / \mathrm{kg} / \mathrm{min})$.

volumes were measured with an apical four chamber echo approach. New York Heart Association (NYHA) functional class and diuretic consumption score were also determined. ${ }^{13}$

QUADRICEPS FEMORIS BIOPSY

A surgical biopsy was taken at the time of bypass surgery and before thoracotomy from the vastus lateralis muscle of the right leg. The size of the specimens was 6-14 $\mathrm{mm} \times 4-8 \mathrm{~mm}$. Biopsies, pinned to a polystyrene support to avoid shrinkage, were immediately frozen in liquid nitrogen.

MUSCLE FATIGUABILITY

The subjects were seated in a rigid frame and maximum isometric strength of the quadriceps femoris was measured with a Cybex Orthotron device (Medway, Massachusetts, USA). ${ }^{13}$ Thereafter patients were asked to carry out repeated voluntary contractions during a five minute protocol at $30-40 \%$ of the maximum. After five minutes a maximum contraction was repeated and fatiguability was expressed as per cent of baseline maximum quadriceps strength. ${ }^{1718}$

CONTROL SUBJECTS

Controls were five male patients with no history of cardiovascular disease and with a normal ECG and echocardiogram, who were undergoing orthopaedic surgery for mid-femur fracture. None had hypertension or diabetes. A biopsy of the vastus lateralis was taken at the time of surgery.

ELECTROPHORETIC SEPARATION OF MYOSIN HEAVY CHAINS

The method is an improvement of that published by Carraro and Catani, ${ }^{19}$ and is described in detail by Vescovo and colleagues. ${ }^{11}$ Biopsies were homogenised and solubilised in $2.3 \%$ sodium dodecylsulfate (SDS), $10 \%$ glycerol, $0.5 \%$ 2-mercaptoethanol, and $6.25 \mathrm{mM}$ Tris-HCl, $\mathrm{pH}$ 6.8. Analytical SDS-page was performed on $7 \%$ polyacrylamide slabs with $37.5 \%(\mathrm{vol} / \mathrm{vol})$ glycerol. Identification of individual myosin heavy chains was performed in a separate series of experiments by immunoblotting the gel bands with a panel of monoclonal antibodies against $\mathrm{MHC}_{1}$ (slow isoform), $\mathrm{MHC}_{2 \mathrm{a}}$ (fast oxidative), and $\mathrm{MHC}_{2 \mathrm{~b}}$ (fast glycolytic). ${ }^{11} 16$
ASSESSMENT OF MYOSIN HEAVY CHAIN DISTRIBUTION

The per cent distribution of the three myosin heavy chains was determined by densitometric scan of the stained slab gels. ${ }^{11} 16$ A linear response in terms of electrophoretic band area is attained on densitometry when $0.1-2.0 \mu \mathrm{g}$ of individual myosin heavy chains are analysed. Quantitative densitometry was performed using an internal myosin heavy chain standard with known percentage distribution of the heavy chains. ${ }^{13} 1416$

SINGLE FIBRE CROSS SECTIONAL AREA

We used single fibre cross sectional areas as an index of myofibre atrophy. ${ }^{14}{ }^{16}$ On each muscle a cross cryosection was taken longitudinally to fibre orientation for histological examination, and stained with haematoxylin and eosin. The cross sectional area of fibres was calculated by means of a computerised interactive method using a Zeiss Ibas 2000 instrument, as described by Angelini and colleagues. ${ }^{20}$ The cross sectional area was expressed in $\mu \mathrm{m}^{2}$. On each specimen at least 400 fibres were counted at $20 \times$ magnification and then averaged.

\section{ASSESSMENT OF APOPTOSIS}

In situ DNA nick end labelling

Serial $8 \mu \mathrm{m}$ thick cryosections from the vastus lateralis were cut and collected on polylysine precoated slides. In situ nick end labelling (TUNEL) of fragmented DNA was performed using terminal deoxynucleotidyl transferase $(\mathrm{TdT})$ and fluorescein conjugated nucleotides with the in situ cell death detection kit, POD (Boehringer-Mannheim, Mannheim, Germany), as described in the manufacturer's instructions. Negative control slides were prepared by substituting distilled water from the TdT enzyme and continuing with the staining. Labelled nuclei were identified from the negative nuclei counterstained by Hoechst 33258 and counted after being photographed under a fluorescence microscope at $250 \times$ magnification as described by Vescovo and colleagues $^{14}$ and Sandri and associates. ${ }^{21}$ The total number of TUNEL positive nuclei (muscle nuclei + all other nuclei present in the specimen) was determined by counting all the labelled nuclei present in the whole specimen. Specimen size varied between 22 and $45 \mathrm{~mm}^{2}$. The count was performed blind. The number 

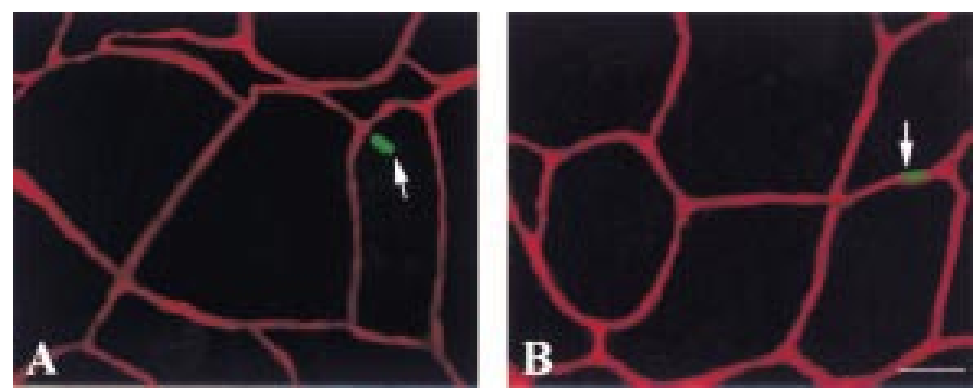

Figure 1 (A) TUNEL positive myocyte nucleus. (B) TUNEL positive interstitial nucleus in the vastus lateralis of a patient with chronic heart failure; the interstitial space is stained with laminin. TUNEL, in situ nick end labelling. Bar $=50 \mu \mathrm{m}$.

of positive nuclei was then expressed as number of apoptotic nuclei $/ \mathrm{mm}^{3}$, taking into account that each specimen was $8 \mu \mathrm{m}$ thick. A separate count was carried out for myofibres and for all the other nuclei, which we called interstitial nuclei-these included fibroblasts, immune cells, and vascular endothelial cells. The myocyte nuclei were distinguished from the interstitial nuclei on the basis of their location on sections stained with laminin, which selectively reacts with the basal lamina. TUNEL positive nuclei that were clearly within the muscle fibre boundary (basal lamina) were counted as myonuclei (fig 1). ${ }^{22}$ All other nuclei were counted as interstitial. It is possible that some of the nuclei may reside in satellite cells, which lie adjacent to the muscle fibres and within the basal lamina. The presence of significant inflammatory infiltrates was excluded on serial histological section stained with haematoxylin and eosin. Separate data for total TUNEL positive nuclei, TUNEL positive myonuclei, and TUNEL positive interstitial nuclei are presented (table 2). Two separate counts were performed by two independent observers and the means reported.

Western blot for Bcl-2, caspase-3, and ubiquitin Fragments of the vastus lateralis were homogenised in SDS buffer. The amount of protein was determined by the Lowry method after precipitation with $10 \%$ trichloroacetic acid. ${ }^{23}$ The homogenates were analysed by SDSPAGE, $12.5 \%$ polyacrylamide, and western blotting by loading $45 \mu \mathrm{g}$ protein/lane. The following conditions were used for binding the antibody: anti-caspase-3 CPP 32 (H-277)

Table 2 Age, myosin heavy chain composition, number of TUNEL positive myocyte and interstitial nuclei, Bcl-2, caspase-3, ubiquitin, and fibre cross sectional area in chronic heart failure (CHF) and control subjects

\begin{tabular}{llll}
\hline & Controls & CHF & p Value \\
\hline Age (years) & $57.1(11.9)$ & $56.8(8.6)$ & NS \\
MHC1 (\%) & $76.0(1.6)$ & $58.4(14.9)$ & 0.0001 \\
MHC2a & $17.8(1.3)$ & $19.8(4.4)$ & 0.0001 \\
MHC2b & $6.2(1.5)$ & $20.7(12.8)$ & 0.0013 \\
TUNEL+ nuclei & & & \\
$\quad$ Interstitial & $4.0(5.5)$ & $33.7(25.9)$ & 0.03 \\
$\quad$ Myocyte & $0.0(0.0)$ & $18.8(19.6)$ & 0.021 \\
$\quad$ Total (n/mm $\left.{ }^{3}\right)$ & $4.0(5.5)$ & $52.4(50.8)$ & 0.015 \\
Bcl-2 (AU) & $101.2(1.8)$ & $65.3(26.7)$ & 0.0001 \\
Caspase-3 (AU) & $151.2(1.65)$ & $453.6(160.8)$ & 0.0001 \\
Ubiquitin $(\mathrm{AU})$ & $133.9(6.0)$ & $157.5(31.4)$ & 0.0001 \\
Fibre CSA $\left(\mu \mathrm{m}^{2}\right)$ & $4415(129)$ & $3768(1024)$ & 0.01 \\
\hline
\end{tabular}

Values are mean (SD)

$\mathrm{AU}$, arbitrary units; CSA, cross sectional area; $\mathrm{MCH}$, myosin heavy chain; TUNEL, in situ nick end labelling.
(34 kDa) and anti-Bcl-2 (29 kDa) (Santa Cruz Biotechnology, Santa Cruz, California, USA) were diluted 1:200 and 1:100, respectively; anti-ubiquitin and anti-rabbit alkaline phosphatase linked antibody (Sigma Chemical Co, St Louis, Missouri, USA) were diluted 1:100 and 1:4000, respectively. The absolute values were calculated on the blot bands with the same densitometric system described above. Values are expressed in arbitrary units (AU) (integrated area of densitometric scans). Bcl-2, caspase-3, and ubiquitin were assessed in all the biopsies. ${ }^{14}$ For caspase-3, we analysed the band derived from the reaction between the caspase- 3 antibody and the activated $20 \mathrm{kDa}$ subunit.

\section{STATISTICAL ANALYSIS}

Mean (SD) values are given. Student's $t$ test for unpaired data was used where appropriate. Linear regression was also used. A 5\% difference was considered significant.

\section{Results}

PATIENT CHARACTERISTICS

Patient characteristics are summarised in table 1.

The mean peak $\mathrm{VO}_{2}$ in the patient group was $12.9(1.7) \mathrm{ml} / \mathrm{kg} / \mathrm{min}$. Fatiguability, measured as per cent of initial maximum strength after five minutes of exercise, was 70.2 (8.8).

COMPARISON BETWEEN PATIENTS WITH CHRONIC HEART FAILURE AND CONTROL SUBJECTS

These results are shown in table 2 .

Age

Age was not significantly different between chronic heart failure patients and controls, at $57.1(11.9)$ v $56.8(8.6)$ years (NS).

\section{Myosin heavy chain composition}

Heart failure patients had a significant decrease in the slow $\mathrm{MHC}_{1}(58.4$ (14.9)\% $v 76.0$ $(1.6) \% ; p<0.0001)$ and an increase in the fast oxidative $\mathrm{MHC}_{2 \mathrm{a}}(19.8$ (4.4)\% v 17.8 (1.3)\%; $\mathrm{p}<0.0001)$ and the fast glycolytic $\mathrm{MHC}_{2 \mathrm{~b}}$ (20.7 (12.8)\% v 6.2 (1.5)\%; p < 0.0013).

\section{TUNEL positive apoptotic nuclei}

In the vastus lateralis of the patients with chronic heart failure there was a significantly greater number of TUNEL positive myocyte and interstitial nuclei (fig 1). The numbers of apoptotic myocyte nuclei were 18.8 (19.6) in the chronic heart failure group versus $0(0)$ in the controls $(p<0.021)$, while the numbers of interstitial apoptotic nuclei were 33.7 (25.9) $v$ 4.0 (5.5) ( $\mathrm{p}<0.03)$. Because of their intralaminin position and their relation to the capillary wall, the majority of the positive interstitial nuclei could be identified as endothelial cell nuclei.

\section{Bcl-2, caspase-3, and ubiquitin}

Caspase- 3 was increased in the vastus lateralis of the heart failure patients $(453.6$ (160.8) $v$ 151.2 (1.65) AU, p < 0.0001), while Bcl-2 was decreased (65.3 (26.7) v 101.2 (1.8) AU, $\mathrm{p}<0.0001)$. Ubiquitin was slightly, though 
A

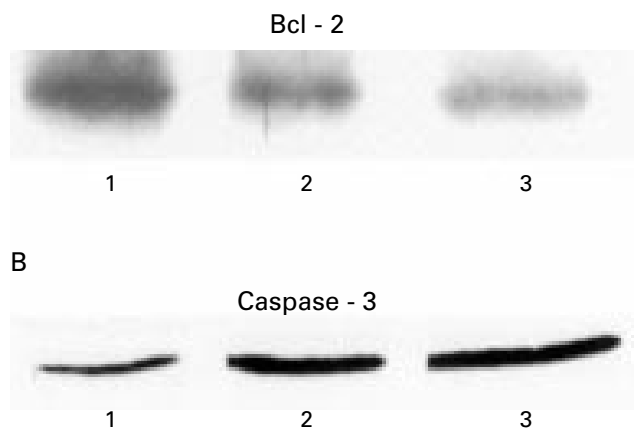

Figure 2 Representative western blot of Bcl-2 (panel A) and caspase-3 (panel B). Lane 1, control; lanes 2 and 3, chronic heart failure patients.

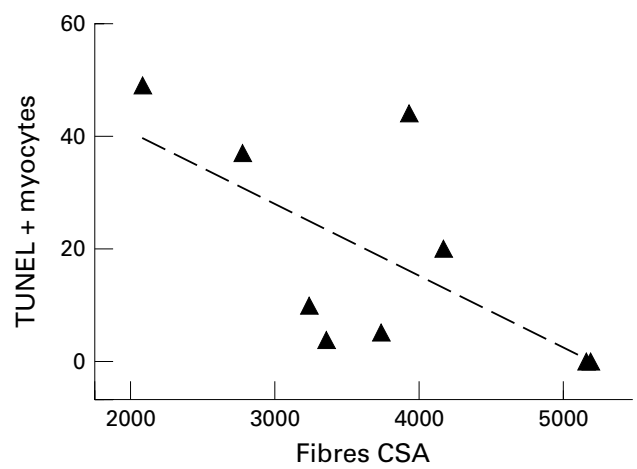

Figure 3 Linear regression between number of TUNEL positive myonuclei/ $\mathrm{mm}^{3}$ and fibre cross sectional area (expressed as $\mu^{2}$ ) in the chronic heart failure patients $(n=9, p=0.05)$. TUNEL, in situ nick end labelling.

significantly, increased as well (157.5 (31.4) v 133.9 (6.0) AU, p < 0.0001). An example of western blot for Bcl-2 and caspase- 3 is shown in fig 2 .

Fibre cross sectional area

Vastus lateralis muscle from the chronic heart failure patients showed a significantly decreased fibre size (3748 (1024) v 4415 (129) $\left.\mu \mathrm{m}^{2}, \mathrm{p}<0.01\right)$.

CORRELATION BETWEEN APOPTOSIS, DEGREE OF FIBRE ATROPHY, MYOSIN HEAVY CHAIN PATTERN, AND INDICES OF SEVERITY OF CHRONIC HEART FAILURE AND FATIGUABILITY

Apoptosis v fibre cross sectional area

We found a negative correlation between number of TUNEL positive myocyte nuclei and fibre cross sectional area $\left(r^{2}=0.51\right.$, $\mathrm{p}=0.004)$. When the data were analysed only within the heart failure group this correlation could still be found $\left(r^{2}=0.44, \mathrm{p}=0.05\right)$ (fig 3).

Apoptosis versus myosin heavy chain composition Although there was a trend for $\mathrm{MHC}_{1}$ to be negatively correlated, and $\mathrm{MHC}_{2 \mathrm{a}}$ and $\mathrm{MHC}_{2 \mathrm{~b}}$ to be positively correlated, with the number of TUNEL positive nuclei, this did not reach significance $\left(r^{2}=0.14,0.16,0.11\right.$ for $\mathrm{MHC}_{1}$, $0.05,0.04,0.06$ for $\mathrm{MHC}_{2 \mathrm{a}}$, and $0.17,0.19$, 0.13 for $\mathrm{MHC}_{2 \mathrm{~b}}$ (total, myocyte, and interstitial nuclei, respectively)).

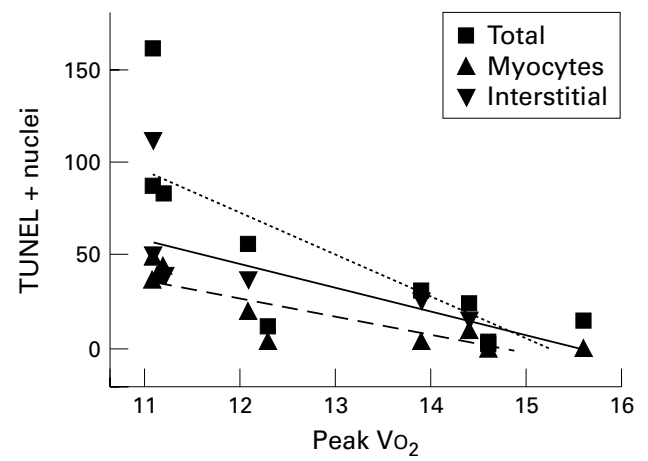

Figure 4 Linear regression between number of TUNEL positive nuclei $/ \mathrm{mm}^{3}$ (total, myocyte, and interstitial) and peak $\mathrm{VO}_{2}$ in the chronic heart failure patients ( $\mathrm{ml} / \mathrm{kg} / \mathrm{min}$ ) $(n=9 ; p=0.03$ for total, $p=0.006$ for myocyte, and $p=0.08$ for interstitial cells).

Apoptosis and myosin heavy chains versus indices of severity of chronic heart failure

There was a correlation between peak $\mathrm{VO}_{2}$ and the number of TUNEL positive nuclei $\left(r^{2}=0.56, \quad \mathrm{p}=0.03\right.$ for total, $r^{2}=0.73$, $\mathrm{p}=0.006$ for myocytes, and $r^{2}=0.41$, $\mathrm{p}=0.08$ for interstitial cells) (fig 4). No significant correlation was found between TUNEL positive nuclei, NYHA class, ejection fraction, diuretic score, and ventricular volumes.

We could not find any significant correlation between Bcl-2, ubiquitin, and caspase- 3 and any of the indices of severity of chronic heart failure (peak $\mathrm{VO}_{2}$, NYHA class, diuretic score, ejection fraction, and ventricular volumes).

Although there was a trend for peak $\mathrm{VO}_{2}$ to be positively correlated with $\mathrm{MHC}_{1}\left(r^{2}=0.16\right.$, $\mathrm{p}=0.5)$ and negatively with $\mathrm{MHC}_{2 \mathrm{a}}\left(r^{2}=0.11\right.$, $\mathrm{p}=0.4)$ and $\mathrm{MHC}_{2 \mathrm{~b}}\left(r^{2}=0.13, \mathrm{p}=0.38\right)$, this did not reach significance. The same was true for NYHA class $\left(r^{2}=0.2\right.$ for $\left.\mathrm{MHC}_{1}, \mathrm{p}=0.2\right)$. No significant correlation was found between myosin heavy chains and ejection fraction, ventricular volumes, and diuretic score. The percentage distribution of myosin heavy chains was not correlated with fibre cross sectional area $\left(r^{2}=0.02, r^{2}=0.00, r^{2}=0.11\right)$. There was, however, a significantly positive correlation between peak $\mathrm{VO}_{2}$ and fibre cross sectional area $\left(\mathrm{n}=9, r^{2}=0.48, \mathrm{p}=0.03\right)$. Furthermore there was a positive correlation between quadriceps femoris fatiguability, peak $\mathrm{Vo}_{2}$ $\left(r^{2}=0.48, \mathrm{p}=0.038\right)$, fibre cross sectional area $\left(r^{2}=0.44, \mathrm{p}=0.048\right)$, and myocyte apoptosis $\left(r^{2}=0.45, \mathrm{p}=0.049\right)$.

\section{Discussion}

It is well known that in chronic heart failure central haemodynamic variables such as cardiac output, ejection fraction, and ventricular volumes and pressures do not correlate with the reduction in exercise capacity. ${ }^{24}$

The early appearance of muscle fatigue is the result of a reduced oxidative capacity, which is closely linked to changes in fibre type and myosin heavy chain composition ${ }^{7-12}$ and to the muscle bulk loss. The relative contribution of these muscle abnormalities to the decreased exercise tolerance and muscle endurance has not been fully elucidated yet. Moreover, little is 
known about the pathogenesis of muscle atrophy. In this paper we have investigated the role played by atrophy in reducing muscle endurance and exercise capacity. Exercise capacity is expressed in term of peak $\mathrm{Vo}_{2}$, which is certainly one of the most precise indices of exercise tolerance. ${ }^{2526}$ At the same time we tested the hypothesis that apoptosis could produce muscle atrophy.

It has been shown by Volterrani and colleagues that the best predictors of exercise capacity in chronic heart failure are measures of skeletal muscle function and bulk. ${ }^{17}$ These investigators have reported that muscle strength and cross sectional area explain $82 \%$ of the variation in peak $\mathrm{VO}_{2}$. Anker and colleagues also showed that there was a correlation between measures of fatiguability and peak $\mathrm{VO}_{2} \cdot{ }^{18}$ However, the link between changes in muscle mass, strength, endurance, and fibre type, and their relation with exercise capacity are still debated.

In this paper we have shown, in according with Adams and colleagues, ${ }^{27}$ that apoptosis occurs in the skeletal muscle of patients with chronic heart failure to a higher degree than in subjects with no evidence of cardiovascular disease, in whom this phenomenon is almost absent. However, in the present paper, we have for the first time tried to determine whether this biological phenomenon is associated with some of the known pathophysiological and clinical features of chronic heart failure. This is a novelty in that previous work on apoptosis in chronic heart failure has been purely observational. ${ }^{28}{ }^{29}$ We have shown that patients with severely limited exercise capacity - as demonstrated by the very low peak $\mathrm{VO}_{2}$ and high fatiguability - have muscle fibre atrophy and increased numbers of myocytes and interstitial nuclei undergoing apoptosis. Because of the relation between the number of apoptotic nuclei and the degree of fibre atrophy, it can be speculated that apoptosis may play a role in determining muscle bulk loss. This is in accordance with some recently published data showing that, in a rat model of heart failure, skeletal muscle apoptosis is accompanied by the occurrence of muscle atrophy. ${ }^{14}$ This is also in keeping with data showing that apoptosis plays an important role in the pathophysiology of chronic heart failure and cardiomyopathies, contributing to myocardial cells loss and therefore to the appearance or worsening of ventricular dysfunction. ${ }^{28} 29$ The only difference between the myocardium and skeletal muscle is that skeletal myocytes are multinucleated; therefore loss of nuclei produces atrophy rather than cell death. ${ }^{22} 30$

In our study the magnitude of apoptosis correlated negatively with peak $\mathrm{VO}_{2}$. This is not surprising as the more symptomatic the patient, the greater are the skeletal muscle abnormalities. The reduction in peak $\mathrm{VO}_{2}$ and endurance correlates with an index of muscle atrophy - the fibre cross sectional area. On the other hand we confirmed in this study an observation made in an experimental model of chronic heart failure, where it was found that levels of apoptosis correlated with the severity of decompensation. ${ }^{14}$ The occurrence of apoptosis in the vastus lateralis muscle of our patients is confirmed by changes in the tissue concentrations of specific factors that can protect from or induce programmed cell death. Bcl-2, which is known to play a protective role, is in fact decreased, and caspase-3, which is a compulsory step in the formation of the apoptotic bodies, is significantly increased. ${ }^{25}{ }^{31}$ The increased ubiquitin concentrations indicate that the proteosome pathway is also activated, leading to protein waste, which may contribute to atrophy independently of apoptosis. ${ }^{32}$

The magnitude of apoptosis is greater in interstitial cells than in myocytes. This has been shown both by our group in experimental chronic heart failure ${ }^{14}$ and by others in skeletal muscle affected by dystrophinopathies. ${ }^{21}{ }^{30}$ The magnitude of apoptosis in our observations is comparable to that found in other studies, the total number $/ \mathrm{mm}^{3}$ of apoptotic nuclei being similar. ${ }^{1422} 33$

At the same time, in the vastus lateralis a significant shift toward the expression of "fast" fatiguable anaerobic fibres occurred, as shown by the increase of the $\mathrm{MHC}_{2 \mathrm{a}}$ and $\mathrm{MHC}_{2 \mathrm{~b}}$ isoforms. Myosin heavy chain redistribution is not likely to be the result of myocyte apoptosis or selective type I fibre apoptosis. ${ }^{14}{ }^{34}$ Our findings confirm previous reports in which patients with chronic heart failure were found to have greater expression of $\mathrm{MHC}_{2 \mathrm{~b}}$ and a decreased percentage of $\mathrm{MHC}_{1} \cdot{ }^{9-13}$ This shift is known to contribute to the limitation of exercise capacity in patients with chronic heart failure. A correlation between peak $\mathrm{VO}_{2}$ and $\mathrm{MHC}_{1}$ has in fact been described. In the present study we only found a trend for peak $\mathrm{VO}_{2}$ to correlate with $\mathrm{MHC}_{1}$, but our study was conducted in severely sick patients $(11.1 \mathrm{ml} / \mathrm{kg} / \mathrm{min} \leqslant$ $\mathrm{VO}_{2} \leqslant 15.6 \mathrm{ml} / \mathrm{kg} / \mathrm{min}$ ), while previous correlations between exercise capacity and $\mathrm{MHC}_{1}$ have been obtained across a wide range of chronic heart failure severity, from asymptomatic early ventricular dysfunction to patients on the waiting list for cardiac transplantation. ${ }^{8-13}$

In the present study exercise capacity seems to have been more influenced by skeletal muscle trophism than by myosin heavy chain composition, as peak $\mathrm{VO}_{2}$ correlated with muscle fibre cross sectional area, while the correlation between peak $\mathrm{VO}_{2}$ and myosin heavy chains was weak. Moreover, it looks as though myosin heavy chain composition and muscle trophism contribute independently to the limitation of exercise capacity, as there was no statistical correlation between these two factors.

Our present data confirm previous observations made by our group in the gastrocnemius of patients with chronic heart failure, in whom the myosin heavy chain shift was neither correlated with muscle mass nor with muscle strength. ${ }^{13}$

The influence of muscle strength and fatiguability on exercise capacity remains controversial, and it has been suggested that some of the reported differences may reflect the varying disease severity in the patients investigated, who therefore presented different degrees of 
muscle atrophy. ${ }^{191718}$ In fact, while in this study - which only included patients with severe chronic heart failure-we found a correlation between indices of muscle trophism (cross sectional area), fatiguability, and peak $\mathrm{VO}_{2}$, in a paper previously published by our group and looking at mild to moderate chronic heart failure, exercise capacity was only marginally dependent on muscle bulk. ${ }^{13}$ However, muscle bulk was estimated in terms of muscle cross sectional area calculated on computed tomography scan slices, which is less sensitive than the measurement of single fibre cross sectional area. The findings in our present paper are partially in agreement with those of Mancini and colleagues, ${ }^{35}$ who found skeletal muscle atrophy in $68 \%$ of patients with chronic heart failure and a correlation between peak $\mathrm{VO}_{2}$ and measures of muscle bulk. However, our data are more in accord with those of Volterrani et $a l,{ }^{17}$ Harrington et $a l,,^{36}$ and Anker et $a l,{ }^{18}$ who found that muscle atrophy becomes a major determinant of exercise capacity. The influence of muscle mass in limiting physical work was also shown by Shepard and colleagues in normal subjects ${ }^{37}$ and by Fleg and Lakatta in the elderly. ${ }^{38}$ In this study we therefore confirm that peak $\mathrm{VO}_{2}$ and atrophy are linked. Our data strengthen the link between apoptosis, atrophy, and muscle function, as myocyte apoptosis and fibre cross sectional area correlated with the decreased muscle strength during repetitive exercise. However, this relation may be true for severely sick and deconditioned patients, while in earlier stages of the chronic heart failure syndrome it may not be so evident. It looks as though the patients in the present study, who had severe chronic heart failure, behaved like those with cardiac cachexia, in whom muscle atrophy further contributes to the deterioration in exercise capacity, not only because of the loss of muscle bulk but also because of the decreased strength per unit area. ${ }^{18} 39$

\section{CONCLUSIONS}

Myocyte and interstitial apoptosis occurs in the leg skeletal muscle of patients with chronic heart failure. The limitation in functional capacity reflects two different skeletal muscle dependent factors: muscle atrophy, which accompanies skeletal myocyte apoptosis, and a shift of myosin heavy chains toward fast more fatiguable isoforms that have higher oxygen and ATP consumption and reach the anaerobic threshold earlier. ${ }^{40}$

We thank Dr Stefano Freguja for the collection of muscle biopsies of control subjects and Mr Valerio Gobbo for skilful technical assistance. The work has been supported in part by a grant "Progetto finalizzato" of the "Veneto Region" Italy.

1 Minotti JR, Pillay P, Chang L, et al. Neurophysiological assessment of skeletal muscle fatigue in patients with congestive heart failure. Circulation 1992;86:903-8.

2 Harridge SPR, Magnusson G, Gordon A. Skeletal muscle contractile characteristics and fatigue resistance in patients with chronic heart failure. Eur Heart f 1996;17:896-901.

3 Wiener DH, Fink LI, Jones RA, et al. Abnormal skeletal muscle bioenergetics during exercise in patients with heart failure: role of reduced muscle blood flow. Circulation 1986;73:1127-36.

4 Arnolda R, Conway M, Dolecki M, et al. Skeletal muscle metabolism in heart failure: a ${ }^{31} \mathrm{P}$ nuclear magnetic resonance spectroscopy study of leg muscle. Clin Sci 1990; 79:583-9.
5 Mancini DM, Ferraro N, Tuchler M, et al. Detection of abnormal calf muscle metabolism in patients with heart failure using phosphorus-31 nuclear magnetic resonance. Am f Cardiol 1988;62:1234-40

6 Massie BM, Conway M, Rajagopalan B. Skeletal muscle metabolism during exercise under ischemic conditions in congestive heart failure: evidence for abnormalities unrelated to blood flow. Circulation 1988;78:320-6.

7 Coats AJS, Clark AL, Piepoli M, et al. Symptoms and quality of life in heart failure. The muscle hypothesis. $\mathrm{Br}$ Heart f 1994;72:36-9.

8 Wilson JR, Fink LI, Maris J. Evaluation of energy metabolism in skeletal muscle of patients with heart failure with gated phosphorus-31 nuclear magnetic resonance. Circulation 1986;71:57-62.

9 Lipkin DP, Jones DA, Round JM, et al. Abnormalities of skeletal muscle in patients with chronic heart failure. Int $\mathcal{F}$ Cardiol 1988;18:187-95.

10 Sullivan MJ, Green HJ, Cobb FR. Skeletal muscle biochemistry and histology in ambulatory patients with long-term heart failure. Circulation 1990;81:518-27.

11 Vescovo G, Serafini F, Facchin L, et al. Specific changes in skeletal muscle myosin heavy chain composition in cardiac failure. Differences with disuse atrophy as assessed on
microbiopsies by a new electrophoretic micromethod. microbiopsies by a $1996 ; 76: 337-43$.

12 Vescovo G, Serafini F, Dalla Libera, et al. Skeletal muscle myosin heavy chain composition in CHF. Correlation between the magnitude of the isoenzymatic shift, exercise capacity and gas exchange measurements. Am Heart $f$ 1998;135:130-7.

13 Vescovo G, Dalla Libera L, Serafini F, et al. Improved exercise tolerance after losartan and enalapril in heart failure: correlation with changes in skeletal muscle myosin heavy chain composition. Circulation 1998;98:1742-9.

14 Vescovo G, Zennaro R, Sandri M, et al. Apoptosis of skeletal muscle myofibers and interstitial cells in experimental heart failure. F Mol Cell Cardiol 1998;30:2449-59.

15 Simonini A, Long CS, Dudley GA, et al. Heart failure in rats causes changes in skeletal muscle morphology and gene expression that are not explained by reduced activity. Circ Res 1996;79:128-36.

16 Vescovo G, Ceconi C, Bernocchi P, et al. Skeletal muscle myosin heavy chain expression in rats with monocrotalineinduced cardiac hypertrophy and failure. Relation to blood flow and degree of muscle atrophy. Cardiovasc Res 1998, 39:233-41.

17 Volterrani M, Clark Al, Ludman PF, et al. Predictors of exercise capacity in chronic heart failure. Eur Heart $\mathcal{f}$ 1994,15:801-9.

18 Anker SD, Swan JW, Volterrani M, et al. The influence of muscle mass, strength, fatigability and blood flow on exercise capacity in cachectic and non cachectic patients with chronic heart failure. Eur Heart f 1997;18:259-69.

19 Carraro U, Catani C. A sensitive SDS-page method separating myosin heavy chains isoforms of rat skeletal muscle reveals the heterogeneous nature of the embryonic myosin. Biochem Biophys Res Commun 1983;116:793-802.

20 Angelini A, Vescovo G, Calliari I, et al. Correlation between endomyocardial biopsies and ventricle full thickness samples in dilated cardiomyopathy: a study of myocytes samples in dilated cardiomyopathy: a study
and fibrosis. Cardiovasc Pathol 1993;3:167-71.

21 Sandri M, Carraro U, Podhorska-Okolov M, et al. Apoptosis, DNA damage and ubiquitin expression in normal and mdx muscle fibers after exercise. FEBS Lett 1995;373:2915.

22 Allen DL, Linderman JK, Roy RR, et al. Apoptosis: a mechanism contributing to remodeling of skeletal muscle in response to hindlimb unweighting. Am f Physiol (Cell Physiol) 1997;273:C579-87.

23 Lowry OH. Protein measure with the Folin phenol reagent. f Biol Chem 1951;195:265-75.

24 Weber KT, Wilson JR, Janicki J, et al. Exercise testing in the evaluation of the patient with chronic cardiac failure. Am Rev Respir Dis 1984;29:60-2.

25 Franciosa A, Park M, Levine T. Lack of correlation between exercise capacity and indexes of resting left ventricular performance in heart failure. Am $\mathcal{F}$ Cardiol 1981;47:33-9

26 Fink L, Wilson R, Ferraro N. Exercise ventilation and pulmonary wedge pressure in chronic stable congestive heart failure. Am $\mathcal{F}$ Cardiol 1986;57:249-53.

27 Adams V, Jiang H, Yu J, et al. Apoptosis in skeletal myocytes of patients with chronic heart failure is associated with exercise intolerance. F Am Coll Cardiol 1999;33:959-65.

28 Narula J, Haider N, Virmani R, et al. Apoptosis in myocytes in end-stage heart failure. $N$ Engl f Med 1996;335:1182-9.

29 Olivetti G, Abbi R, Quaini F, et al. Apoptosis in the failing human heart. N Engl f Med 1997;336:1131-41.

30 Sandri M, Podhorska-Okolow M, Geromel V, et al. Exercise induces myonuclear ubiquitination and apoptosis in dystrophin-deficient muscle of mice. $\mathcal{F}$ Neuropathol Exp Neurol 1997;56:45-57.

31 Steller H. Mechanisms and genes of cellular suicide. Science 1995;267:1445-9.

32 Llovera M, Garcia Martinez C, Agell N, et al. TNF can directly induce the expression of ubiquitin-dependent proteolytic system in rat soleus muscles. Biochem Biophys Res Commun 1997;230:238-41.

33 Tews DS, Goebel HH, Schneider I, et al. DNAfragmentation and expression of apoptosis related proteins in experimentally denervated and reinnervated facial muscle. Neuropathol Appl Neurobiol 1997;23:141-9. 
34 Dalla Libera L, Zennaro R, Sandri $M$ et al. Apoptosis and atrophy in rat slow skeletal muscles in chronic heart failure. Am f Physiol (Cell Physiol) 1999;277:C982-6.

35 Mancini DM, Walter G, Reichek N, et al. Contribution of skeletal muscle atrophy to exercise intolerance and altered muscle metabolism in heart failure. Circulation 1992;85: 1364-73.

36 Harrington D, Anker SD, Chua TP, et al. Skeletal muscle function and its relation to exercise tolerance in chronic heart failure. Am $\mathcal{F}$ Coll Cardiol 1997;30:1758-64.
37 Shepard R, Bouhlel E, Vandewalle H. Muscle mass as a factor limiting physical work. F Appl Physiol 1988;64: $1472-9$

38 Fleg J, Lakatta E. Role of muscle loss in the age-associated reduction in $\mathrm{VO}_{2}$ max. F Appl Physiol 1988;65:1147-51.

39 Anker SD, Coats AJS. Cachexia in heart failure is bad for you. Eur Heart $\mathcal{F}$ 1998;19:191-3.

40 Ruegg JC. In: Calcium in muscle contraction, 2nd ed. Berlin: Springer Verlag, 1992:125.

\section{IMAGES IN CARDIOLOGY}

\section{Prolapse of an atrial myxoma into the apex of the left ventricle}

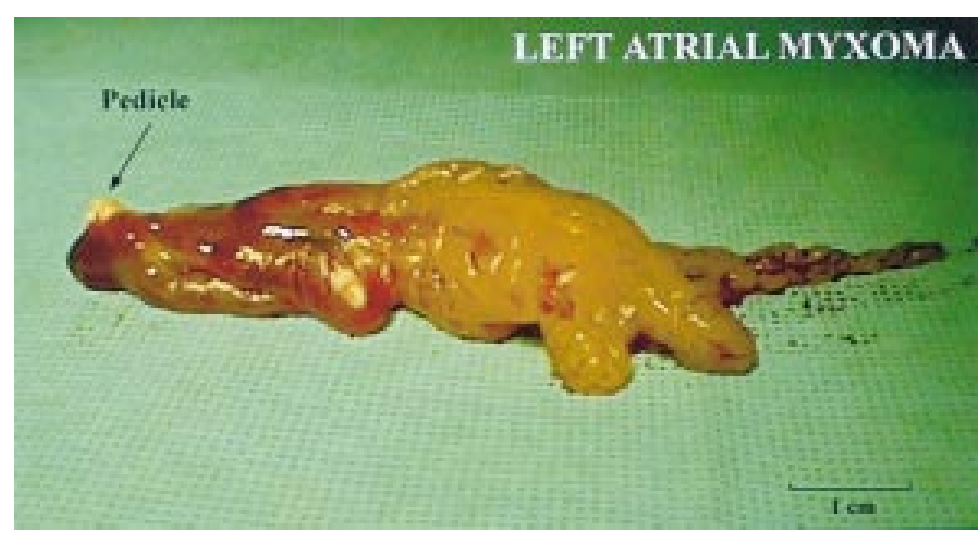

WEB EXTRA: access eHeart to see this move; there is a link from the table of contents for this issue on the Heart web site (www.heartjnl.com)
A 31 year old scuba diving instructor presented with a history of recurrent palpitations while diving to depths of $30 \mathrm{~m}$. An early diastolic sound was heard on cardiac auscultation. Transthoracic and transoesophageal echocardiography demonstrated a large left atrial mass, attached to the interatrial septum, which prolapsed into the left ventricle during diastole. Surgical exploration of the left atrium revealed a $7.5 \times 1.9 \times 0.9 \mathrm{~cm}$ polypoid, gelatinous tumour arising from the fossa ovalis via a narrow pedicle. The lesion was excised in its entirety and subsequent histology confirmed the clinical diagnosis of left atrial myxoma. Following surgery the patient remains well.

C S R BAKER

P STRIKE A DEANER 